\title{
SPATIAL ANALYSIS IN PUBLIC HEALTH DOMAIN: AN NLP APPROACH
}

\author{
Pattathal Vijayakumar Arun \\ College of Science and Technology, Phuentsholing, Bhutan \\ E-mail:arunpv2601@gmail.com
}

Received 10 May 2013; accepted 09 December 2013

\begin{abstract}
Remote sensing products are effectively used as a tool for decision making in various fields, especially in medical research and health care analyses. GIS is particularly well suited in this context because of its spatial analysis and display capabilities. The integration of RS techniques in public health has been categorised as continuous and discrete strategies where latter is preferred. We have investigated the integration of these approaches through linguistic interpretation of images. In this paper, we propose a framework for direct natural language interpretation of satellite images using probabilistic grammar rules in conjunction with evolutionary computing techniques. Spectral and spatial information has been dynamically combined using adaptive kernel strategy for effective representation of the contextual knowledge. The developed methodology has been evaluated in different querying contexts and investigations revealed that considerable success has been achieved with the procedure. The methodology has also demonstrated to be effective in intelligent interpolation, automatic interpretation as well as attribute, topology, proximity, and semantic analyses.
\end{abstract}

Keywords: NLP, remote sensing, image translation.

Reference to this paper should be made as follows: Arun, P. V. 2013. Spatial analysis in public health domain: an NLP approach, Geodesy and Cartography 39(4): 149-157.

\section{Introduction}

The benefits of remote sensing techniques are being extensively integrated across a range of disciplines, and are enhanced with the economic feasibility and flexibility of Earth Observation products. The synoptic, multi-spectral and multi-temporal coverage provided by EO programs have made it increasingly suitable for analyses in public health field. However these data collected is just a fraction of what could be put to excellent, perhaps life saving use in every region of world. Earth science application in medical field varies from infectious disease mapping to emergency preparedness and response planning (Turker, Sumer 2008). An integrated approach is needed for effective use of RS data, especially in medical research and health care analyses. Rs data requires to be translated to interpretable form before it can be put to effective use.

Remote sensing analysis strategies in this context are generally biased as continuous and discrete, in which former uses pixel based strategy and latter object based. Rather than classifying each pixel based on its spectral content alone, the object based (discrete) approach adopts spatial, spectral and contextual information to segment the features.The increased availability of high resolution images has enhanced the use of object based approaches; however continuous products such as NDVI are still effectively used in various critical analyses. For example discrete mapping may be used to capture vector habitat and other health exposures where as land cover analyses requires a continuous strategy. Kelly et al. (2011) suggested that the depiction of geographic object in a discrete sense is more useful and accurate than pixel based approaches in various analyses. However Cohen et al. (2010) have found the continuous approach as useful for malaria analysis using land use/land cover and demographic data. Maxwell's works in this context highlights that spatial fidelity as well as improved accuracy makes object based approaches preferable. Literature reveals that performance of both the approaches is situation specific and needs to be integrated for an effective analysis (Addink et al. 2009; Ebert et al. 2011; Blaschke, Hay 2011; Kelly et al. 2004). Resolution of imagery as 
well as the required parameter should be considered for selecting effective strategy (Liu, Weng 2009; Graham et al. 2005).

Direct natural language translation of RS imagery will not only facilitate the integration of continuous and discrete strategies but also effective spatial mining. This further enhances the integration of spatial data with linguistic non spatial public health data. Effective mining is affected by the lack of a general approach over image and non image data. Direct querying of image data will enhance the utility of remote sensing products for effective decision making. Interpretation of images in natural language form will facilitate effective retrieval, analysis and mining of image data. The efficiency of spatial analysis in public health domain has been affected by the poor image analysis expertise of physicians and can be tackled using semi automatic linguistic translation of visual data. Advances in deduction and mining techniques over the language domain can be effectively integrated with various aspects of computer vision. Semantic queries in image aspects require integrated visual as well as linguistic analysis that can be accomplished through effective visual translation. Specific approaches are found over the literature where as a generalized integrated attempt is less explored (Graham et al. 2005; Bhaskaran et al. 2010; Dambach et al. 2011).

We explore the linguistic translation of images for integrating continuous and discrete image interpretation strategies; thereby providing a generalized decision support frame work for public health domain. This frame work provides an NLP query interface to the user and image analyses can be done as in a text document. We have investigated the feasibility of integrating NLP and evolutionary computing approaches for automatic linguistic interpretation of spatial data. Integration of computer vision and Natural Language Processing (NLP) techniques in the remote sensing context has been less explored, except for a few relevant general approaches (Zhu, Mumford 2006; Zhu et al. 2010; Siskind et al. 2007; Socher et al. 2012). Our studies have found that inverse mapping of Cellular Automata (CA) using Genetic Algorithm (GA) can be adopted for effective modelling of feature shapes (Orovas, Austin 1998; Mitchell et al. 1996). Spectral and spatial information has been combined using an adaptive kernel strategy to improve effectiveness of the approach. PCFG based rule sets in conjunction with evolutionary computing techniques is found to be effective for contextual rule representation. The proposed framework enables interpretation of images using natural language, and hence facilitates automation of various image interpretation tasks.

In this paper, we present a framework for automatic parsing of spatial data to natural language descriptions, so as to aid the public health decisions. Thus we propose a linguistic translation of image data for integrating discrete and continuous image interpretation approaches. Automatic object modelling, adaptive kernel mapping, automatic interpretation, topology mapping, parameter estimation, auto learning and intelligent interpolation are salient features of this work. Accuracy of the framework has been evaluated in different querying contexts with reference to various satellite images.

\section{Theoretical background}

\subsection{Random modelling techniques}

Evolutionary computing approaches such as CA, GA and their variants such as Cellular Neural Network $(\mathrm{CNN})$ and Multiple Attractor Cellular Automata (MACA), have been found to be useful for modelling random features. CNN (Orovas, Austin 1998; Mitchel et al. 1996) is effectively used for modelling object shape to facilitate feature interpretation. Random rules governing the shape of a feature can be identified by evolving the feature from a single state using $\mathrm{CNN}$ and GA. Abstract representations of objects are obtained by evolving features continuously until they can be separated from the background. MACA is a special type of CA with different local rules applied to different cells and will converge to certain attractor states on execution (Sikdar et al. 2000). MACA is initialized with an unknown pattern and operated for a maximum (depth) number of cycles until it converges to an attractor. PEF bits after convergence are extracted to identify the class of the pattern and are compared with stored rules to interpret the object. Thus these random modelling techniques are effectively used for modelling various objects and interpreting them.

\section{2. $\mathrm{N}$-dimensional classifiers}

N-Dimensional classifiers such as Support vector are non-probabilistic binary linear classifiers that constructs a set of hyperplanes to optimally separate the classes. SVRF (Schnitzspan et al. 2008; Lee et al. 2005) is a Discrete Random Field (DRF) based extension for SVM. It considers interactions in the labels of adjacent data points while preserving the same 
appealing generalization properties as the Support Vector Machine (SVM). SVRF is used along with the kernel functions to implement initial clustering for accurate detection and interpretation. Kernel functions are used along with SVRF approaches to increase the dimensionality of the classification space. They measure the similarity between two data points that are embedded in a high, possibly infinite, dimensional feature space. Mixture Density Kernel (MDK) measures the number of times an ensemble agrees that two points arise from same mode of probability density function (Srivastava 2004). Mixture density kernels are used to integrate an adaptive kernel strategy to the SVRF based clustering as they facilitate learning of kernels directly from image data rather than using a static approach.

\subsection{Coreset}

Coreset (Agarwal et al. 2001; Badoiu et al. 2002) is a small subset of a point set, which is used to compute a solution that approximates solution of the entire set. Let $\mu$ be a measure function (e.g., width of a point set) from subsets of $\mathrm{Rd}$ to non-negative reals $\mathrm{R}+\mathrm{U}\{0\}$ that is monotone, i.e., for P1 C P2, $\mu(\mathrm{P} 1) \leq \mu(\mathrm{P} 2)$. Given a parameter $\varepsilon>0$, we call a subset $\mathrm{Q} C \mathrm{P}$ as an $\varepsilon$-Coreset of $\mathrm{P}$ (with respect to $\mu$ ) if $(1-\varepsilon) \mu(\mathrm{P}) \leq \mu(\mathrm{Q})$. Coreset optimisation can be adopted to reduce the number of pixels required to represent an object by preserving its shape. Hence it can be used to reduce the complexity of CA based inverse evolution.

\subsection{N LP Parser}

NLP parsers detect the syntactic structure of sentences with reference to a defined grammar, for instance, parsers may identify phrases, subjects, objects, verbs etc. Probabilistic parsers use knowledge of language gained from hand-parsed sentences to try to produce the most likely analysis of new sentences. We have used a Stanford parser which is a java implementation of probabilistic natural language parsers, namely lexicalized dependency parser and lexicalized PCFG parser. The lexicalized probabilistic parser implements a factored product model, with separate PCFG phrase structure and lexical dependency experts, whose preferences are combined by efficient exact inference, using an $\mathrm{A}^{*}$ algorithm (de Marneffe et al. 2006). NLP Parser along with WordNet has been used to interpret the queries and to infer the attribute requirement. A PCFG grammar based rule set has been adopted to estimate the required parameters for a particular object and are dynamically updated.

\subsection{WordNet}

WordNet provides a lexical database for the English language. It groups words into sets of synonyms called synsets, provides general definitions, and records semantic relations between these synonym sets (Fellbaum 1998). It serves as a thesaurus that is more intuitively usable, and supports automatic text analysis as well as artificial intelligence applications. WordNet is used for the lexical analyses of queries along with parser based syntactic analyses.

\section{Experiment}

\subsection{Dataset description}

Different satellite images of Bhopal and Chandrapur have been used as test images for evaluating the system performance with reference to various queries. Investigations have been conducted over various image datasets namely LISS4 \& LISS 3 sensor images of IRS P5 satellites having resolution $23.5 \& 5.8 \mathrm{~m}$ respectively. Analysis was also conducted using LANDSAT ( $30 \mathrm{~m}$ resolution) \& Google Earth imageries. The ground truthing information has been collected using a Differential Global Positioning System (DGPS) survey conducted over Bhopal and Chandrapur during October and November 2012 respectively. System has been also experimented using the real time datasets from NIMHANS hospital, Bangalore, India.

\subsection{Methodology}

A schematic representation of the adopted methodology is presented in Figure 1. We have restricted the queries with reference to public health domain; however the approach can be further generalized. The initial data collected from patient is submitted to the system to collect the relevant formation. The query is processed to get location as well as parameter information, and related imageries are acquired from the openly available spatial providers such as USGS and Google Earth. Recently developed spatial web standards (e.g. Web Mapping Service (WMS), Web Coverage Service (WCS), Web Feature Service (WFS), Web Terrain Service (WTS), Geographic Markup Language (GML), etc.) are adopted to implement the crawling. The common features that required to be analyzed are categorized using a decision tree and further physicians are provided with provisions to specify additional features.

Different image features are detected using $\mathrm{CNN}$ based shape modeling approaches and are further interpreted using MACA based pattern detection. 


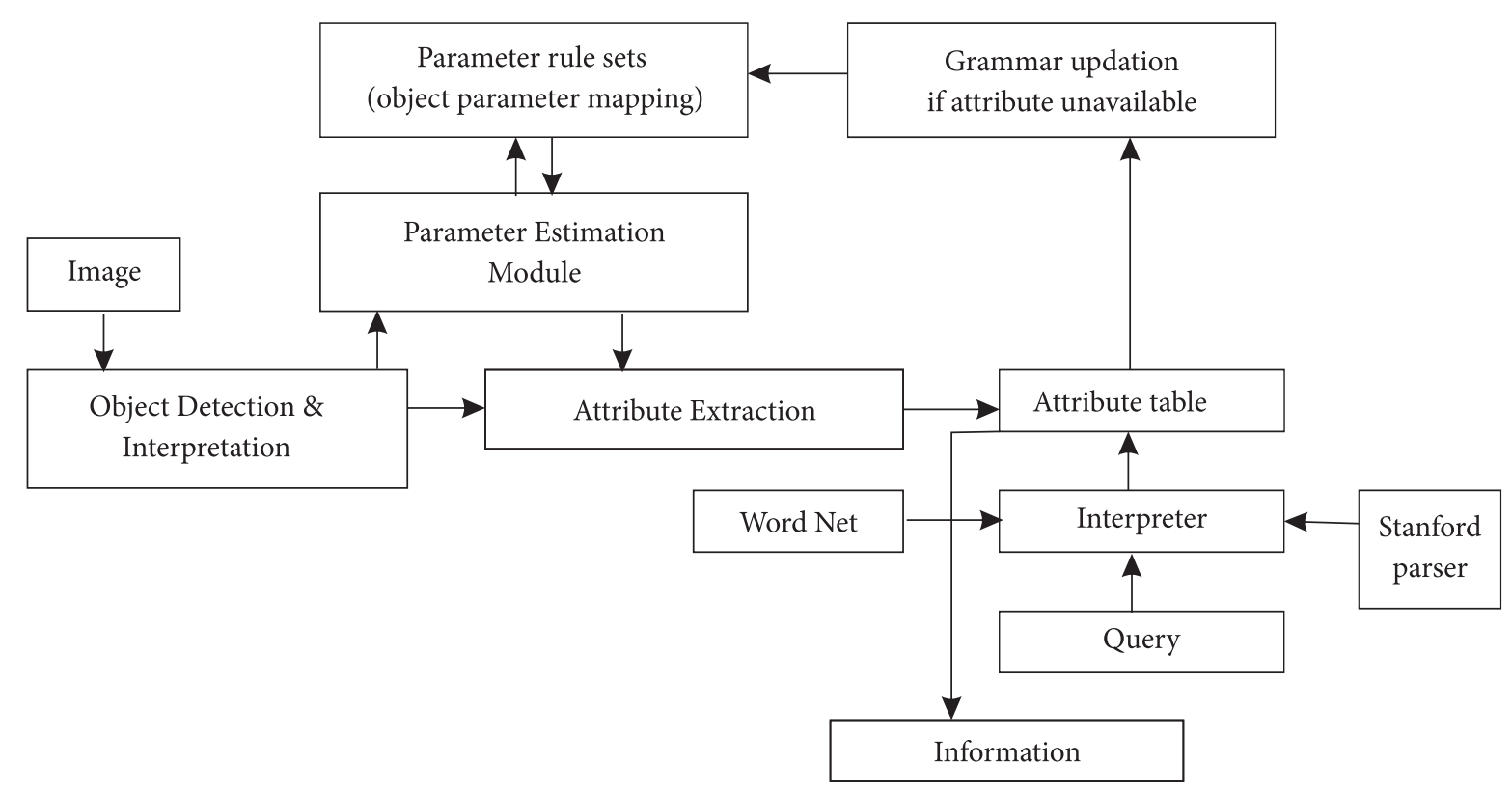

Fig. 1. Methodology adopted

Parameters associated with each image feature are estimated based on general rules (Probabilistic Context Free Grammar), and are extracted to corresponding attribute tables. Queries are interpreted using a Stanford parser-WordNet interface and required attributes are fetched from the table. If an attribute is unavailable, parameter estimation rules are automatically updated to associate that attribute with the corresponding object. Detailed descriptions of the different steps are given below.

\section{Object extraction}

Abstract representation of image features is initially obtained using edge detectors along with the CA based region growing strategy. The image is then clustered using a mixture density kernel based SVRF approach, and the process is enhanced using abstract object information. Parameters of mixture density kernels are adjusted automatically based on ensembles, and are exploited to incoporate contextual information as well as the adaptive kernel strategy. Detected objects along with boundary information are optimized using the corset approach to reduce the complexity of shape modeling. Clustered objects along with edge information are utilized to model feature shapes using $\mathrm{CNN}$ and MACA. Inverse mapping of $\mathrm{CNN}$ is exploited for the purpose, and $\mathrm{CNN}$ rules used to evolve a particular feature are used to distinguish it. Rules corresponding to various features are thus deducted and are mapped in a prolog DB. Detected objects are further interpreted using shape-rule mapping that maps objects to corresponding MACA rules. Interpolation of features such as roads and rivers is accomplished using CA rules integrated with stored predicate rules. Planning related to various health activities requires understanding of spatial change in various realms such as land cover, drainages, urban growth etc and is accomplished using the corresponding feature information over different temporal image datasets.

\section{Attribute extraction}

Spatial attributes required for health analyses are extracted automatically based on linguistic queries and extracted data is easily integrated to decision support systems. Image metadata, along with the feature information, is used to extract the object attributes. Probabilistic Context Free Grammar (PCFG) based rule sets are used to determine the attributes required for each object. Identified parameters are extracted to corresponding object tables and are used as attributes to provide the required image description. Available coordinate information as well as auxiliary data is also used as attributes to provide topological as well as proximity information.

\section{Topology interpretation}

PCFG rule sets are used to govern the topology extraction and relative positions are determined based on the coordinate information associated with each feature. Comparisons of boundary pixel positions are adopted for determining relative positions of random features. A relative rectangular co-ordinate system is assumed for images if exact coordinate information is not available. 
Topology information, along with simple spatial buffering, is adopted to process the proximity queries.

\section{Visualization}

Visualization queries are accomplished using the imageries along with required elevation details and facilitates effective real time decisions. The queries are automatically interpreted, and available datasets are used to provide required modeling. DEMs and other images are automatically extracted from openly available sources such as SRTM, ASTER, and USGS, based on location information provided in the query. Effective spatial visualization is also provided to facilitate real time decisions. Buffering can be effectively used to track the spatial extent of a particular disease and to check the probable impact. The vaccination as well as other health tasks requires proper planning and hence zonation as well as buffering may serve the purpose. Random modelling approaches will help the analyzer to predict spread pattern of disease and will help in planning effective counter measures. The effect of pollution as well as environmental factors are predicted and located by adopting spatial modelling approaches such as CA, regression analyses along with other statistical tools.

\section{NLP interface}

Stanford parser, along with WordNet, is used to process the NLP queries and required information is fetched from corresponding object attribute tables. Queries are lexically analyzed using WordNet and syntactically analysed using Stanford parser. Querying regarding an unavailable attribute is accomplished by seeking user interaction, and the parameter estimation grammar is revised to include the attribute along with its calculation methodologies. Thus a dynamic learning strategy is adopted to automatically improve the extraction grammar.

\section{Results and discussions}

Investigations over the proposed framework using various satellite images revealed that considerable success have been achieved with the procedure. The system has been evaluated in different querying contexts and found successful over various datasets. Linguistic queries were accurately interpreted to identify the object, and related attributes have been further deduced using the probabilistic rules. Different parameters such as drainage patterns, water sources, urban settlement etc are critical for various health related analysis like pollution exposure modelling, risk analysis, flood modelling, social vulnerability mapping etc. Mapping of resident area and urban structures is required for various health analysis like risk mapping, vaccination mapping, disease spread analysis, zoning etc. Object extraction is effective only over high resolution imageries and hence continuous approach is adopted for land cover detection over coarser imageries. System adopts continuous or discrete strategies based on the parameters as well as the availability of imageries. Disease spread and related analysis requires various minute features and their attributes different from usual approach. Hence the integration of discrete and continuous strategies in a linguistic frame work seems to be effective.

Initial investigations have been conducted over features specific queries concerning various objects. These queries facilitate user to extract a particular feature, for example user can query to extract a river from the imagery. The extraction of water bodies as well as land cover features from PAN and LANDSAT images of study area is shown in Figure 2.

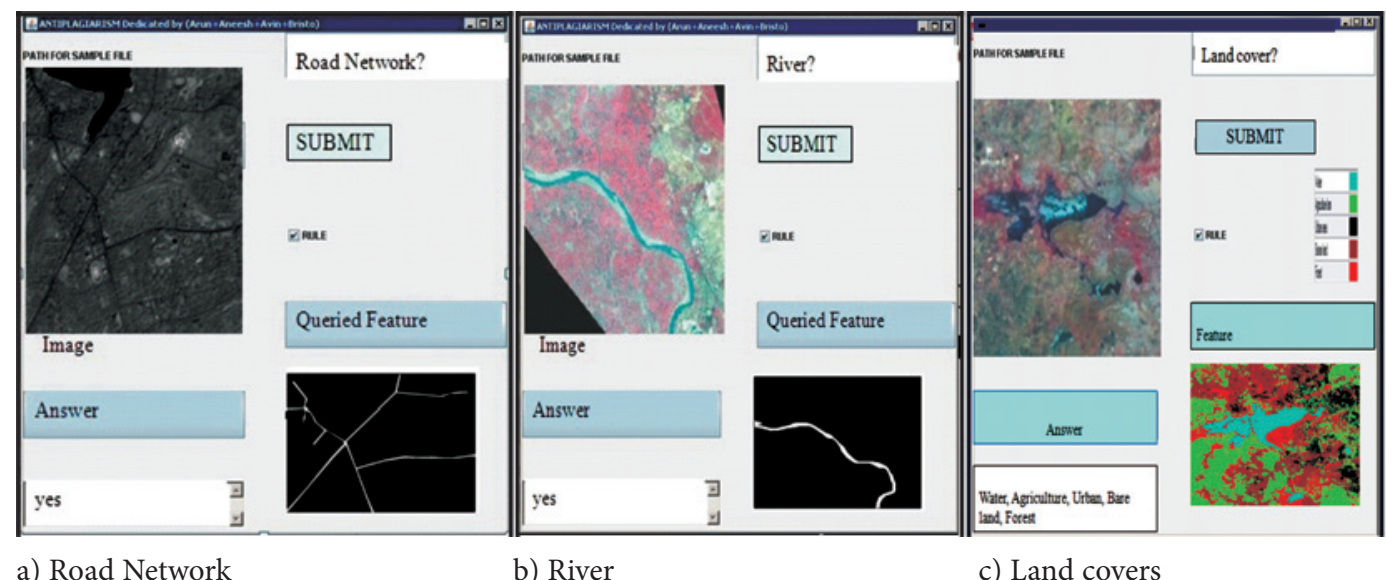

a) Road Network

b) River

c) Land covers

Fig. 2. Feature extraction queries 


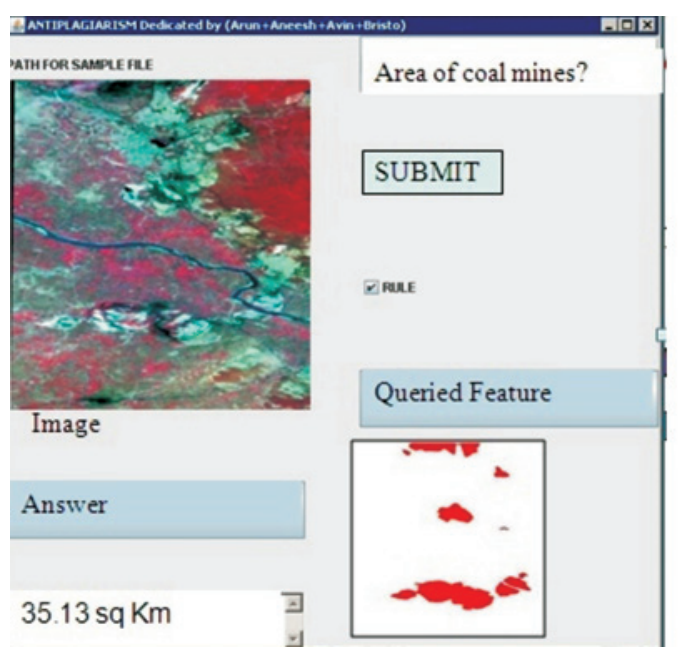

a) Coal mines

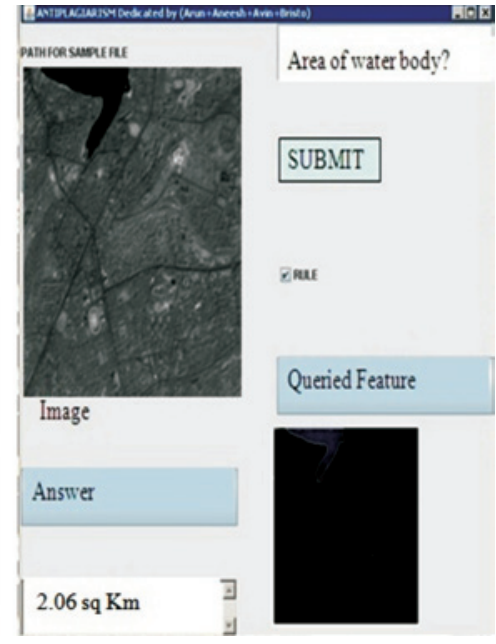

b) water body

Fig. 3. Attribute specific queries

Table 1. Performance summary for advanced queries

\begin{tabular}{|c|c|c|c|}
\hline $\begin{array}{l}\text { S. } \\
\text { No } \\
\end{array}$ & Sensor & Query Type & $\begin{array}{c}\text { Average } \\
\text { accuracy (\%) }\end{array}$ \\
\hline \multirow{7}{*}{1} & \multirow{7}{*}{ LISS 3} & Feature extraction Queries & 91.38 \\
\hline & & $\begin{array}{l}\text { Attribute specific Queries } \\
\text { (Feature Area) }\end{array}$ & 89.37 \\
\hline & & Position specific Queries & 88.10 \\
\hline & & Resolution specific Queries & 93.13 \\
\hline & & Proximity Queries & 85.70 \\
\hline & & Topology Queries & 82.29 \\
\hline & & Numbering Features & 90.13 \\
\hline \multirow{7}{*}{2} & \multirow{7}{*}{ LISS 4} & Feature extraction Queries & 98.91 \\
\hline & & $\begin{array}{l}\text { Attribute specific Queries } \\
\text { (Feature Area) }\end{array}$ & 94.21 \\
\hline & & Position specific Queries & 96.10 \\
\hline & & Resolution specific Queries & 98.23 \\
\hline & & Proximity Queries & 93.89 \\
\hline & & Topology Queries & 88.45 \\
\hline & & Numbering Features & 96.76 \\
\hline \multirow{7}{*}{3} & \multirow{7}{*}{$\begin{array}{l}\text { LAND- } \\
\text { SAT- TM }\end{array}$} & Feature extraction Queries & 85.32 \\
\hline & & $\begin{array}{l}\text { Attribute specific Queries } \\
\text { (Feature Area) }\end{array}$ & 78.12 \\
\hline & & Position specific Queries & 81.20 \\
\hline & & Resolution specific Queries & 88.19 \\
\hline & & Proximity Queries & 71.97 \\
\hline & & Topology Queries & 65.25 \\
\hline & & Numbering Features & 82.90 \\
\hline \multirow{7}{*}{4} & \multirow{7}{*}{$\begin{array}{l}\text { Google } \\
\text { Earth }\end{array}$} & Feature extraction Queries & 99.96 \\
\hline & & $\begin{array}{l}\text { Attribute specific Queries } \\
\text { (Feature Area) }\end{array}$ & 94.83 \\
\hline & & Position specific Queries & 98.10 \\
\hline & & Resolution specific Queries & 97.52 \\
\hline & & Proximity Queries & 96.81 \\
\hline & & Topology Queries & 84.45 \\
\hline & & Numbering Features & 98.59 \\
\hline
\end{tabular}

The pixel based approach has been applied to extract the land cover features (Fig. 2c) and system intelligently selects the discrete or continuous strategy based on image resolution as well as feature types. Figure 2 shows effective integration of continuous and discrete strategies for feature queries. The efficiencies of these queries have been evaluated with reference to various statistical parameters such as over all accuracy and kappa statistics (MacLean, Congalton 2011; Congalton 1991). These parameters have been computed using confusion matrices by considering each object as a separate class. Average efficiency of the system for feature queries over various datasets is summarized in Table 1 . These results reveal that system accurately extracts features over various data sets. Higher detection accuracies over LISS 4 and Google earth imageries may be attributed to their high resolution.

System also enables user to query about various feature attributes, for example user can query about area, perimeter, distance etc of various features. This further enables user to have comparative queries over different features. Figures $3 \mathrm{a}$ and $3 \mathrm{~b}$ shows the area extraction queries in which areas of coal mines and water bodies are queried respectively.

System accurately extracts various attributes and this enables different analyses such as change detection, spatial mining, impact analysis etc. Queries concerning feature areas have been cross verified for various features, namely lakes, coal mines, and parks; since these features have well defined and fixed geometry. Original surface areas of various extracted features have been calculated by manual digitization using ERDAS software, and average accuracy of extraction has 
been analyzed. The average accuracy of areal extent queries over various datasets is presented in Table 1.

Performances of the system have also been evaluated with reference to co-ordinate or position specific queries and were verified using DGPS survey. These types of queries include those concerning the position of a feature and are answered by utilizing the coordinate information (derived from metadata). Determination of effective position of random features is a problem in this context and is usually calculated based on boundary pixel co-ordinates. Raster (Pixel) coordinate systems are automatically assigned to estimate relative positions in case if geographic coordinates are not available. Comparative analysis system performance for position specific queries over various datasets is summarized in Table 1.

Position data in conjunction with feature information is used to answer various context specific queries. Proximity information of features are deduced by utilizing appropriate buffering approach and is used to find features within a certain distance. Figure 4 shows a simple 3D visualisation to locate areas in the vicinity of a coalmine which can be utilized to analyze the effect of pollution. Average accuracy of proximity queries over various data sets is summarized in Table 1 .

The above information (feature, proximity, attributes etc) along with metadata are used for implementing advanced queries such as those concerning feature counts, resolution, topology etc. A summary of the comparative analysis of system performance over these queries is presented in Table 1. Ground truthing has been used to evaluate the effectiveness of system with reference to these queries. Google earth and DGPS survey over the study areas using Trimble R3 DGPS equipment has been conducted for the purpose.

Results from these analyses indicate that the framework has been successful in dealing with different categories of spatial queries. Different features are automatically interpreted and their attributes are estimated in accordance with the probabilistic rules. These attributes along with contextual information and metadata are used to provide an effective description as well as visualization of the image. The various automatic elucidations as discussed above are integrated to provide accurate image descriptions. Thus system successfully parses images to a natural language description as shown in Figure 5.

The description query as shown in Figure 4 automatically extracts the feature data, attribute description and also sensor specific information. These data are further used to provide effective visualization and deduction. Geo spatial visualization is implemented using openly available DEM along with satellite images of corresponding regions. Figure 6 shows areas that

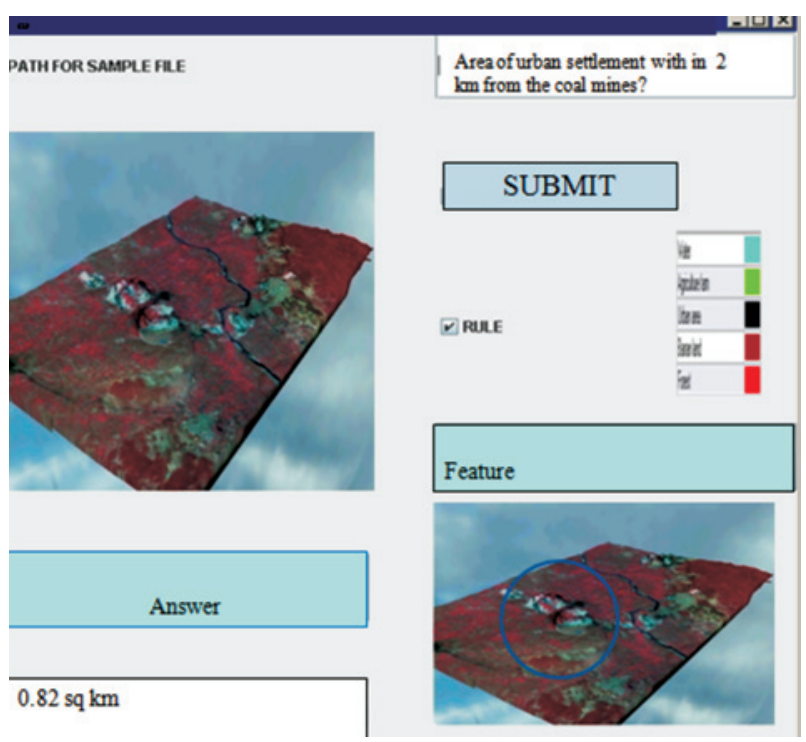

Fig. 4. Proximity queries

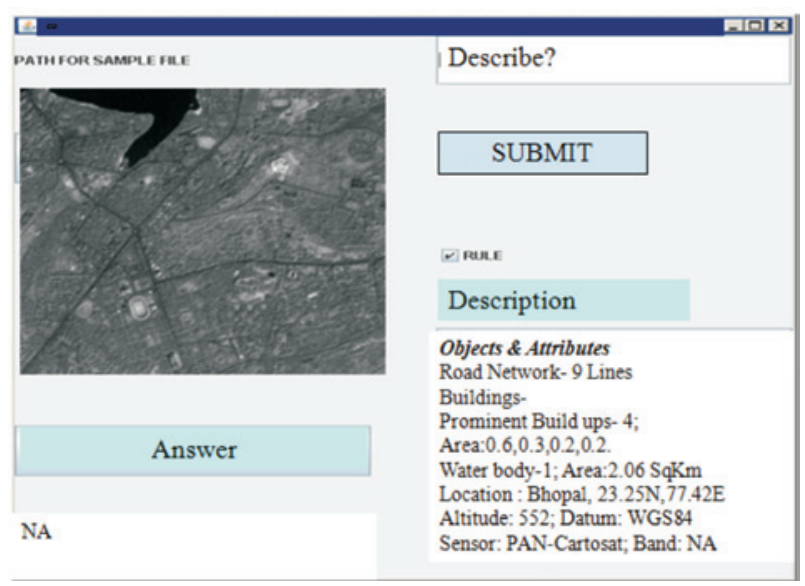

Fig. 5. Description queries

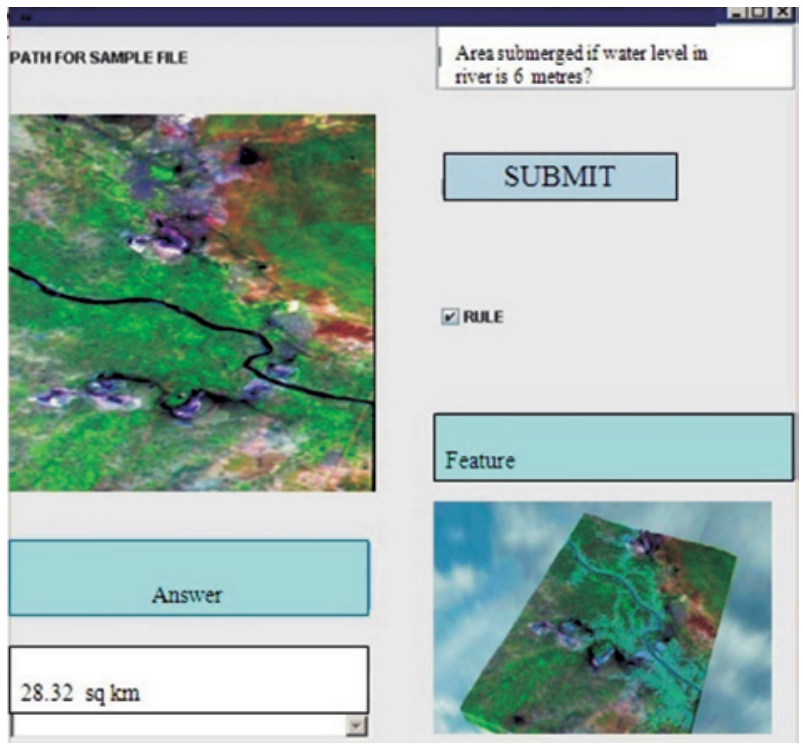

Fig. 6. Visualization queries 
will be flooded if river water rises to a particular level. As evident these visualizations can be effectively used by the medical practitioners as well as authorities for better analysis or effective planning.

Thus the frame work enables to interpret the images directly through natural language or in other words accomplishes linguistic translation of a visual scene. The approach can be used in various applications and enhances the decision making capability as it allows direct mining of spatial data. This approach also facilitates the direct integration of linguistic techniques with computer vision approaches.

Parameter estimation of certain features, such as roads, requires a semi automatic approach for detection rather than a complete automatic method. The developed method was found to be less effective to describe complex topological queries as evident from Table 1 where the accuracy is comparatively less. This may be attributed to the difficulty in defining relative positions of random features. Effective interpolation of feeble road networks also requires manual interpretation. The main disadvantage of the method is its computational complexity which can be improved by coreset optimization and similar approximation techniques. Complexity can be further reduced by storing the detected rule variations; optimization methods such as GA can be exploited to optimize the strategy. This research provides a basic framework and further investigations are needed to optimize it. Integration of a fuzzy approach to the inverse mapping also seems to be promising, since fuzzy / neutrosophic cognitive maps can be exploited for effectively organizing and selecting CA rules. The PCFG grammar update approach also needs further improvement especially in the context of topological attributes.

\section{Conclusion}

Remote sensing technique holds distinct promise as a tool in the fight against emerging infectious diseases and other public health problems. Object based approaches in public health domain are found to be more effective than simple pixel based or NDVI based approaches; however a proper integration is desirable. Linguistic interpretations of imageries have proved to be effective in this context. In this research we have discussed a framework for the effective semantic interpretation of images to facilitate direct imaging queries. Frame work has been used for the accurate extraction of various parameters required for disease analysis as well as for modeling various random disease related phenomenon. Investigations have revealed that the method performs effectively in different querying contexts. The proposed framework has proved to be effective with reference to accurate interpolation, and interpretation. The reduction of ambiguity of features, enhanced detection, self learning, minimal human interpretation, and reliability are features of the system. Further investigations are needed over the improvement of the framework, especially on parallelizing and optimizing different operations for complexity reduction. Effective representation of different context rules also needs further improvement, and techniques such as fuzzy cognitive maps seem to be promising in this context. Sequence images, as well as effective topology processing, can also be explored to achieve full utilization of the framework. The framework can be further improved to enable semantic analysis of related image datasets for facilitating effective decisions.

\section{References}

Addink, E. A.; de Jong S. M.; Davis, S. A.; Dubyanskiy, V.; Leirs, H. 2009. Using very high spatial resolution remote sensing to monitor and combat outbreaks of bubonic plague in Kazakhstan, in Proceedings of Anais XIV Simpósio Brasileiro de Sensoriamento Remoto, April, 2009, Natal, Brazil, 7529-7536,

Agarwal, P. K.; Aronov, B.; Sharir, M. 2001. Exact and approximation algorithms for minimum-width cylindrical shells, Discrete Computational Geometry 26(3): 307-320.

Badoiu, M.; Har-Peled, S.; Indyk, P. 2002. Approximate clustering via corsets, in Proceedings of 34th Annual ACM Symposium, Theory of Computation, 19-21 May, 2002, Montréal, Québec, Canada, 250-257.

Bhaskaran, S.; Paramananda, S.; Ramnarayan. M. 2010. Per-pixel and object-oriented classification methods for mapping urban features using Ikonos satellite data, Applied Geography 30: 650-665. http://dx.doi.org/10.1016/j.apgeog.2010.01.009

Blaschke, T.; Hay, G. J. 2011. Object-oriented image analysis and scale-space: theory and methods for modeling and evaluating multiscale landscape structure, in Proceedings of ISPRS WG IV/3, IV/5, IV/6 and IV/7 Workshop "Challenges in GeoSpatial Analysis, Integration and Visualization", 22-29 October, 2011, Athens, GA, USA, vol. 34, no. 4/W5.

Cohen, J. M.; Ernst, K. C.; Lindblade, K. A.; Vulule, J. M.; John, C. C.; Wilson, M. L. 2010. Local topographic wetness indices predict household malaria risk better than land-use and land-cover in the western Kenya highlands, Malaria Journal 9: 328. http://dx.doi.org/10.1186/1475-2875-9-328

Congalton, R. G. 1991. A review of assessing the accuracy of classifications of remotely sensed data, Remote Sensing of Environment 37(1): 35-46.

http://dx.doi.org/10.1016/0034-4257(91)90048-B

Dambach, P.; Sie, A.; Lacaux, J. P.; Vignolles, C. C.; Machault, V.; Sauerborn, R. 2011. Using high spatial resolution remote sensing for risk mapping of malaria occurrence in the Nouna district, Burkina Faso, Journal of Remote Sensing 3: 2343. 
de Marneffe, M.-C.; MacCartney, B.; Manning, Ch. D. 2006. Generating Typed Dependency Parses from Phrase Structure Parses, in Proceedings of 5th International Conference on Language Resources and Evaluation (LREC 2006), 24-26 May, 2006, Genoa, Italy, 449-454.

Ebert, A.; Kerle, N.; Stein, A. 2011. Urban social vulnerability assessment with physical proxies and spatial metrics derived from air-and spaceborne imagery and GIS data, Natural Hazards 48: 275-294.

http://dx.doi.org/10.1007/s11069-008-9264-0

Fellbaum, Ch. 1998. WordNet: an electronic lexical database. Cambridge, MA: MIT Press, 120-125.

Graham, A.; Danson, F.; Craig, P. 2005. Ecological epidemiology: the role of landscape structure in the transmission risk of the fox tapeworm Echinococcus multilocularis, Progress in Physical Geography 29: 77-92. http://dx.doi.org/10.1191/0309133305pp435ra

Kelly, M.; Blanchard, S. D.; Kersten, E.; Koy. K. 2011. Terrestrial remotely sensed imagery in support of public health: new avenues of research using object-based image analysis, $R e$ mote Sensing 3: 11: 2321-2345. http://dx.doi.org/10.3390/rs3112321

Kelly, M.; Shaari, D.; Guo, Q. H.; Liu, D. S. 2004. A comparison of standard and hybrid classifier methods for mapping hardwood mortality in areas affected by sudden oak death, Photogrammetric Engineering and Remote Sensing 70: 12291239. http://dx.doi.org/10.14358/PERS.70.11.1229

Lee, C.; Schmidt, M; Greiner, R.; Support vector random fields for spatial classification, in Proceedings of 9th European Conference on Principles and Practice of Knowledge Discovery in Databases (PKDD), 3-7 October, 2005. Porto, Portugal. 196.

Liu, H.; Weng, Q. 2009. An examination of the effect of landscape pattern, land surface temperature, and socioeconomic conditions on WNV dissemination in Chicago, Environmental Monitoring and Assessment 159: 143-161. http://dx.doi.org/10.1007/s10661-008-0618-6

MacLean, M. G.; Congalton, R. G. 2011. Investigating issues in map accuracy when using an object-based approach to map benthic habitats, GIScience and Remote Sensing 48(4): 457-477. http://dx.doi.org/10.2747/1548-1603.48.4.457

Mitchell, M.; Crutchfield, J. P.; Das, R, 1996. Evolving Cellular Automata with genetic algorithms: a review of recent work, in Proceedings of First International Conference on Evolutionary Computation and Its Applications (EvCA'96), 20-22 May, 1996, Nayoya University, Japan 1(1): 120-130.
Orovas, C.; Austin, J. 1998. A cellular system for pattern recognition using associative neural networks, in Proceedings of IEEE International Workshop on Cellular Neural Networks and their Application, 14-17 April, 1998, Lisbon, 2(4): 11.

Schnitzspan, P.; Mario, F.; Bernt, S. 2008. Hierarchical support vector random fields: joint training to combine local and global features, in Proceedings of 10th European Conference on Computer Vision, 12-18 October, 2008, Palais des Congrès Parc Chanot, Marseille, France 53(5): 527-540.

Sikdar, B. K.; Paul, K.; Biswas, G. P.; Yang, C.; Bopanna, V.; Mukherjee, S.; Chaudhuri, P. P. 2000. Theory and application of $\mathrm{GF}(2 \mathrm{P})$ cellular automata as on-chip test pattern generator, in Proceedings of 13th Int. Conf. on VLSI Design, 4-5 January, 2000, Mumbai, India, 556-561.

Siskind, J. M.; Sherman, J.; Pollak, I.; Harper, M. P.; Bouman, C. A. 2007. Spatial random tree grammars for modeling hierarchal structure in images with regions of arbitrary shape, in Proceedings of IEEE Transactions on Pattern Analysis and Machine Intelligence 29: 120-127.

Socher, R.; Huval, B.; Bhat, B.; Manning, Ch. D.; Ng, A. Y. 2012. Convolutional-recursive deep learning for 3D object classification, Advances in Neural Information Processing Systems 25: $1-9$.

Srivastava, A. N. 2004. Mixture density Mercer Kernels: a method to Learn Kernels directly from data, in Proceedings of 4th SIAM International Conference on Data Mining, 22-24 April, 2004, Lake Buena Vista, Florida, USA.

Turker, M.; Sumer, E. 2008. Building-based damage detection due to earthquake using the watershed segmentation of the post-event aerial images, International Journal of Remote Sensing 29: 3073-3089. http://dx.doi.org/10.1080/01431160701442096

Zhu, L.; Chen, Y.; Torralba, A.; Freeman, W. T.; Yuille, A. L. 2010. Part and appearance sharing: Recursive Compositional Models for multiview, in Proceedings of 23rd IEEE Conference on Computer Vision and Pattern Recognition (CVPR), 13-18 June, 2010, San Francisco, USA, 1234-1244.

Zhu, S. C.; Mumford D. 2006. A stochastic grammar of images, Foundation and Trends in Computer Graphics Visualisation 2(4): 259-362.

Pattathal Vijayakumar ARUN. He has completed his Masters from NIT-Bhopal, and is currently pursuing $\mathrm{PhD}$. His main area of interest are artificial Intelligence, spatial mining, and image processing. 